\title{
Design and Development of Energy Measurement System based on the Android Platform
}

\author{
Rakesh Dwivedi \\ Center of Development of Advanced Technology \\ Mohali, Punjab
}

\begin{abstract}
In late years, smart-devices became very popular. This paper presents an application of power monitoring system using android application, which is convenient and efficient to achieve visualization of real time signals that are received from smart meters via GSM module. This application can be used by the consumer for measurement of electrical usage in the house and control their power usages accordingly. In this the analog data is converted to digital data with the help of inbuilt ADC and passes it to the Microcontroller This application, built with wireless signals to mobile via GSM technology on the Android platform. Android base cell phones itself go about as a transmitter and receiver using ATmega16 microcontroller, power supply and GSM SIM300.
\end{abstract}

\section{Index Terms}

GSM, ATmega16 Microcontroller, Android smart phone, smart meter

\section{INTRODUCTION}

These days, there has been an incredible change in clients' inclination in the mobile sector with the quick headways in chip innovation and programming. More individuals' utilization cell phone for making calls or sending messages, as well as for hobby, for example, social network services, games, music purposes and person to person communication administrations. In a world of exceptionally created nations and developing commercial concerns, power supply assumes a real part. In a cutting edge family, scarcely any gadget runs without power. So the energy consumption and conservation is a vital part of the household.

The traditional electric meter, used by electricity companies for accounting is the electromechanical induction watt-hour meter. Its robust technical design is in use for over a century, but is not capable of more than measuring the accumulated amount of consumed energy [13]. A disadvantage is that either an employee, sent by the energy company or the customer himself has to read the meter manually which implies costs and administration effort

The conventional electric meter, utilized by power organizations for accounting is the electromechanical actuation meter. The electromechanical meter is used only for measuring the collective measure of consuming energy. A detriment is that either a worker, sent by the vitality organization or the client himself need to peruse the meter physically which infers expenses and organization exertion.

The electricity supply company adopts the electronic meters, i.e. Electronic measurement of energy expenditure. These meters have diminished assembling expense, enhanced measurement exactness, precise data and most importantly reduced size, due to these benefits that go past the customary rotor plate energy meter. The smart meter is identified as AMR and AMI technology.
In this paper an application of power measurement on android platform is developed. This application is an online service that connects to consumer's smart phone via GSM technology. Smart meter measures the power consumed and send data to consume smart phone via GSM technology. The main objective behind the development an efficient power measuring application for displaying power consumed on the Android phone. The system was designed to transmit wireless signals directly to the Android Phone via GSM technology on the Android Platform [2]. The consumers can easily visualize the power consumed by then because it does not require any mounted monitor.

\section{ANDROID}

In the field of android operating system, the Android 1.0 was the first Android Operating System, which was commercially released in September 2008.This mobile application development platform was open source. The collaboration of the Google with the Open Handset Alliance was designed this Android Platform. The Android SDK has many tools and different APIS, which can use in designing and developing different mobile apps. Android has growth as a main mobile platform because of its updated and software features [5]. The latest version of the Android was Android L API level 21 which was released on 16 June, 2014 and updated on 25 June, 2014 of Android Kit Kat 4.4.4

Linux is used as the operating system for Android OS. This android OS is used in mobile, smart phone and tablets. The developers can use the full features of the hardware system because it is an open source platform for mobile applications.

The environment of android and the android architecture is a software based which has some parts like the Linux OS, number of application frameworks, different libraries, end user applications and more. Figure 1 shows the architecture of android.

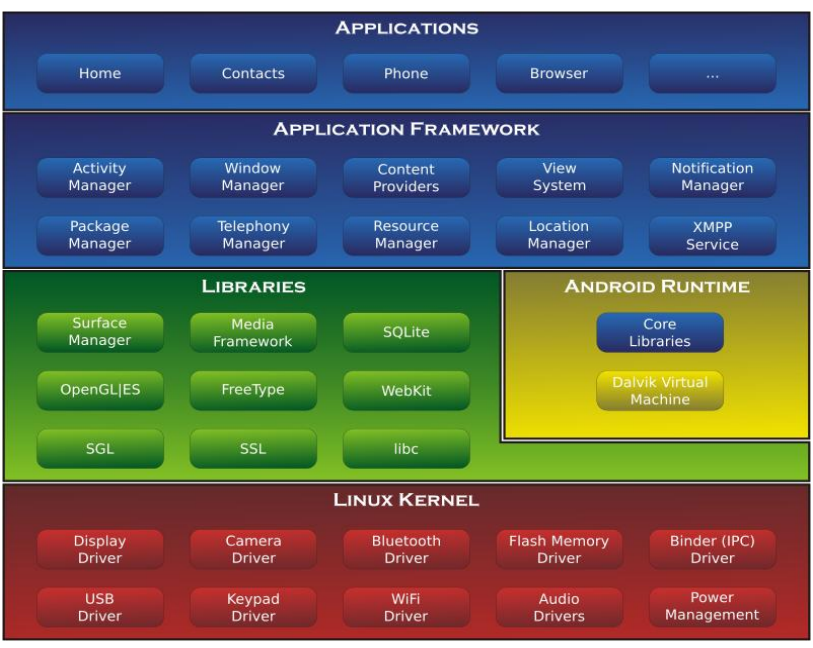

Fig 1: Android Architecture 


\subsection{Linux Kernel}

The bottom layer of android architecture consists of Linux (Linux 2.6) having 115 numbers of patches. This system contains basic functions such as managing a number of processes, memory management, managing a number of devices for e.g. camera, keyboard, display, etc. It's the duty of the kernel which manages all the resources that Linux is an efficient platform such as networking and a vast area of device drivers.

\subsection{Libraries}

Kernel consists the bunch of libraries on the top layer of Linux, which includes open-source like WEB engine, valuable libraries, SQLite database helpful for storage and sharing of data application, libraries which are helpful for playing and recording audios and videos, and other libraries used for internet security purpose.

\subsection{Android Runtime}

It is the third part of the android architecture. This platform provides a precious component known as Dalvik Virtual Machine. It is a sort of Java Virtual Machine used for designing and optimizing for Android systems. The Dalvik VM is helpful to provide various resource management, such as memory management and multi-threading. The DVM makes every application of android to execute in their own process. The Android architecture also consists of libraries which make Android developers to develop Android applications with the help of standard Java programming language.

\subsection{Framework of Application}

This layer gives a lot of high level services to android based application in the form of classes of Java. Developers of applications are having permission to make use of the above mentioned services in their designed applications.

\subsection{Applications}

Top layer consists of all types of Android applications. The users can write their application to be installed on this layer only. Such as like Contact, Books, Browser, Games and many more. Elements of the application are the important components of an Android application.

\section{SYSTEM OVERVIEW}

The block diagram of the system is shown in figure-2. With power supply circuit, supply power to other components. In this The TXD pin of the Microcontroller is connected to the RXD pin of GSM model and vice-versa.

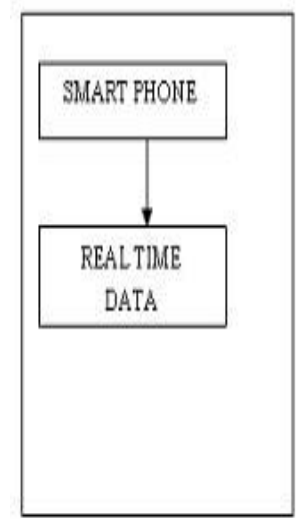

A) RECENER

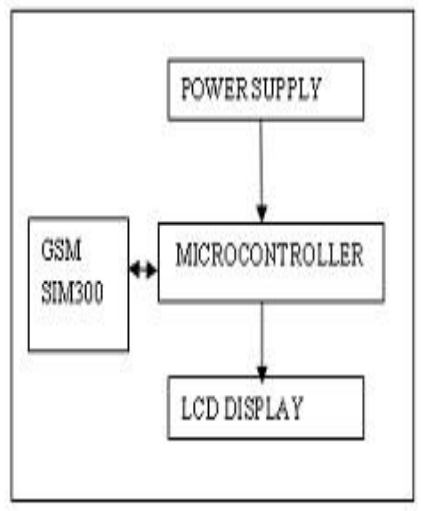

B) TRANSMITTER
The first step is to start the application which represents the introduction of application. Second step describes the GSM Communication between the smart meter and the smart phone. Third step is the user case to read the reading coming from smart meter. Figure 3 shows Flow diagram of the application activity.

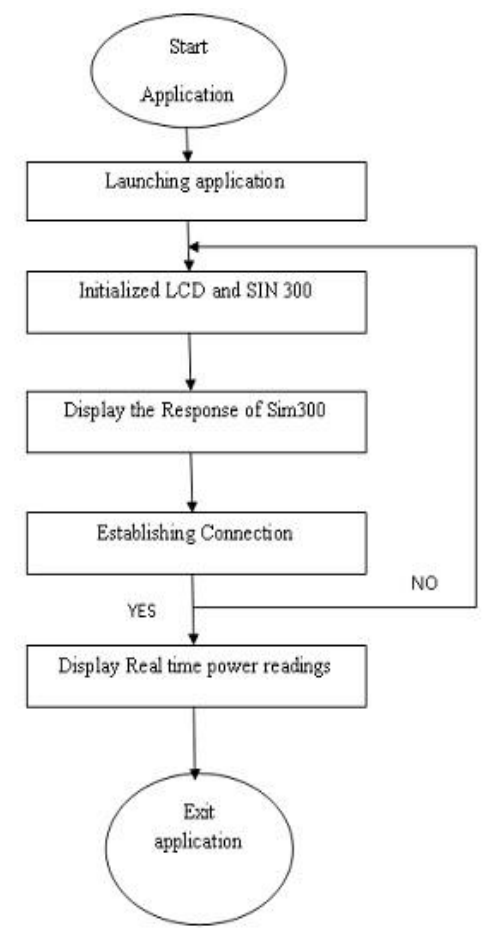

Fig 3: Flow Diagram

\section{IMPLEMENTATION AND RESULTS}

The Wireless android application is to be represented in automatic way which provides the useful functionality to display real time power coming from the smart meter. A Communication medium between the smart meter and the Android phone is GSM Module.Gsm module connection is required to send the real time power. The application code was written in the Java programming language using Eclipse Integrated Development Environment (IDE) with Android Developer Tools (ADT)[3].

Eclipse is the simplest way to create any components or Android project . The Android application is a User's Interface (UI) to the GSM module. This application gives the consumer the option to calculate power,recording, display the data.

Another important file for developing the android application is android manifest. Androidmanifest.xml record is utilized within every android application which is found in the root registry of android undertaking. This will create naturally when you make an android application. The android manifest.xml document contains data of your android application to the android framework, before the application's code run. The androidmanifest.xml document is likewise used to characterize diverse consent, for example, NFC (Near Field Communication), camera access, Bluetooth and so on.

The system hardware for this is shown in figure-4.the components used in the project such as ATmega16 microcontroller, power supply, GSM module and transformer[18].

Fig 2: System Architecture 


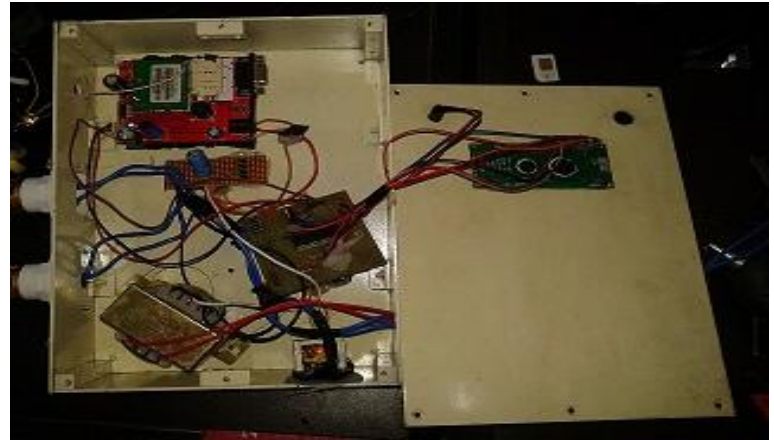

Fig 4: system hardware

The developed Wireless android application is running on the Samsung grand Quattro smart phone which has 4.1.2 jelly bean android operating system. The output of the application is in the form of captured images.

Following images are captured by Samsung grand Quattro android phone: Figure 5 shows that the icon of Wireless android application.

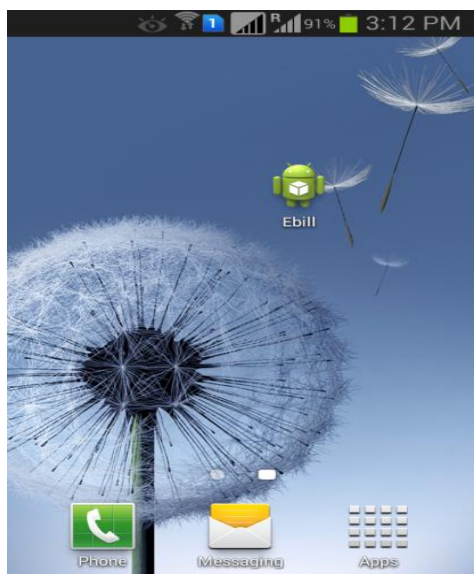

Fig 5: android application

Double click on android icon it opens the login page and after login the main dialog box will open as shown in figure 6 and figure 7 respectively.

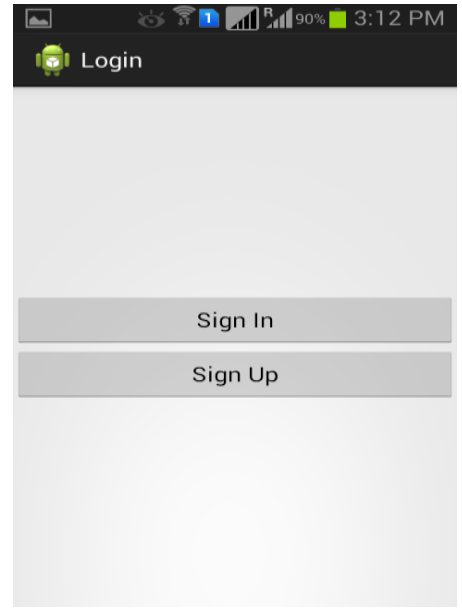

Fig 6: login page

Figure 7 shows that after login to android application the reading from smart meter send to the smart phone via GSM module.

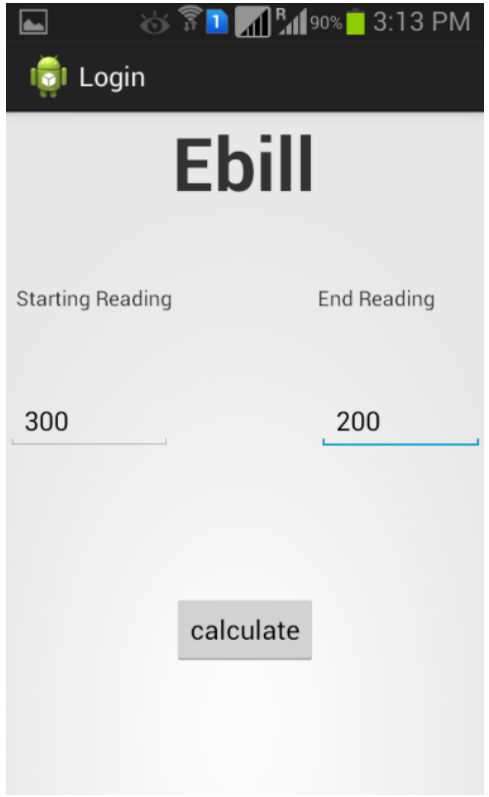

Fig 7: Ebill window
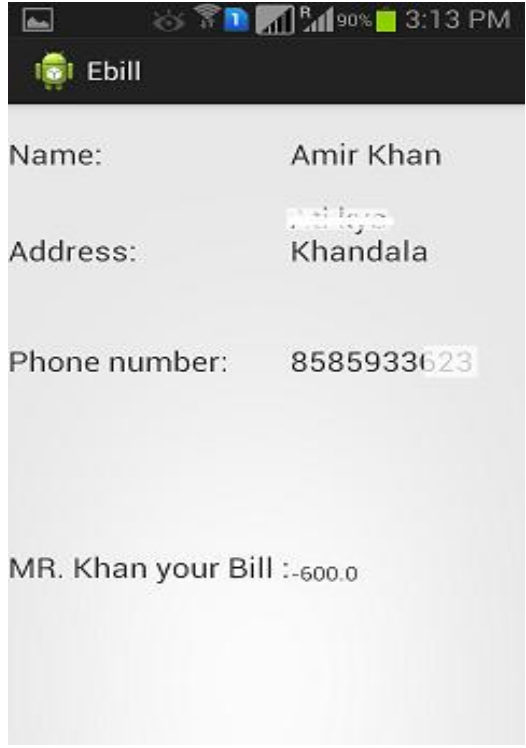

Fig 8: consumer Ebill

The final dialog box will appear after calculation, which contain the consumer name, address and phone number.

\section{CONCLUSION}

In this paper we design and develop an Android application for power measuring using a wireless plug. This system is designed to monitor the energy consumption and send that consumption to the smart phone via a wireless plug. This application runs on the android base smart phone only which support android OS 4.2.1. This system consists of three main parts which include data gathering and data processing and smart phone. After testing the entire above objective are successfully achieved and implemented. We developed a hardware and software solution for monitoring the power consumption. Some limitations are arising in this system such as low sampling rate and low resolution. After this we are capable of combining latest mobile technology with the electrical and wireless technology and developed a simple and robust system for power utility companies. 


\section{REFERENCES}

[1] Dennis Joe Harmah. "An ubiquitous miniaturized android based ECG monitoring system.” International Conference on Emerging Trends in Computing, Communication and Nanotechnology (ICECCN 2013), pp. 117-120. IEEE, 2013.

[2] Bedarkar, Poonam T., and Shanti Swamy. "Design and implementation of wireless handheld device for android cell phone." In Proceedings of the Conference on Advances in Communication and Control Systems 2013, pp. 284-90. 2013.

[3] Liang, Z. H. A. O., and Wei ZHANG. "Research and Design of Interface Based on Android Technology [J]." Computer Knowledge and Technology 29 (2009): 041.

[4] Guang-xian, J. I. "Applied Prospect and Development Research Under the Android System Environment." Software 10 (2011): 018.

[5] Shi-Cheng, Z. H. A. N. G. "Development and Research of Application Based on Google Android." Computer Knowledge and Technology 28 (2009): 051.

[6] Colunas, Márcio FM, José Maria Amaral Fernandes, Ilídio Castro Oliveira, and João P. Silva Cunha. "Droid Jacket: Using an Android based smartphone for team monitoring." In Wireless Communications and Mobile Computing Conference (IWCMC), 2011 7th International, pp. 21572161. IEEE, 2011.

[7] Wagner, Matthias, Benjamin Kuch, Carlos Cabrera, Peter Enoksson, and Arne Sieber. "Android based Body Area Network for the evaluation of medical parameters." In Intelligent Solutions in Embedded Systems (WISES), 2012 Proceedings of the Tenth Workshop on, pp. 33-38. IEEE, 2012.

[8] Yi, Won-Jae, Weidi Jia, and Jafar Saniie. "Mobile sensor data collector using Android smartphone." In Circuits and Systems (MWSCAS), 2012 IEEE 55th International Midwest Symposium on, pp. 956-959. IEEE, 2012.

[9] Lau, Hon Fai, and Shigeru Yamamoto. "Bayesian online changepoint detection to improve transparency in humanmachine interaction systems." In Decision and Control (CDC), 2010 49th IEEE Conference on, pp. 3572-3577. IEEE, 2010.

[10] Bircher, William Lloyd, and Lizy K. John. "Complete system power estimation: A trickle-down approach based on performance events." In Performance Analysis of Systems \& Software, 2007. ISPASS 2007. IEEE International Symposium on, pp. 158-168. IEEE, 2007.
[11] Mahesri, Aqeel, and Vibhore Vardhan. "Power consumption breakdown on a modern laptop." In Poweraware computer systems, pp. 165-180. Springer Berlin Heidelberg, 2005.

[12] Rusitschka, Sebnem, Christoph Gerdes, and Kolja Eger. "A low-cost alternative to smart metering infrastructure based on peer-to-peer technologies." In Energy Market, 2009 EEM 2009. 6th International Conference on the European, pp. 1-6. IEEE, 2009.

[13] Han, Dae-Man, and Jae-Hyun Lim. "Smart home energy management system using IEEE 802.15. 4 and zigbee." Consumer Electronics, IEEE Transactions on 56, no. 3 (2010): 1403-1410.

[14] Singh, Jaspal. "Development Of Automatic Geo-Fencing And Accidental Monitoring System Based On Gps Technology." International Journal of Computer Science, Engineering \& Applications 3, no. 4 (2013).

[15] Moloo, Raj Kishen, and Varun Kumar Digumber. "Lowcost mobile GPS tracking solution." In Business Computing and Global Informatization (BCGIN), 2011 International Conference on, pp. 516-519. IEEE, 2011.

[16] Lian, Jie, Yunhao Liu, Kshirasagar Naik, and Lei Chen. "Virtual surrounding face geocasting in wireless ad hoc and sensor networks." Networking, IEEE/ACM Transactions on 17, no. 1 (2009): 200-211.

[17] M. Faisal and A. Mohamed, "A new technique for power quality based condition monitoring," in 17th Conf. Electrical Power Supply Industry, Oct. 2008. A. Al-Yasiri and A. Sunley, Data aggregation in wireless sensor networks using the SOAP protocol," in J. Physics: Conf. Series 76012039, 2007

[18] M. Choi, S. Ju, and Y. Lim, "Design of integrated meter reading system based on power line communication," in IEEE International Symp. Power Line Commun. Its Appl., Apr. 2008.

[19] Gurun, Selim, and Chandra Krintz. "A run-time, feedbackbased energy estimation model for embedded devices." In Proceedings of the 4th international conference on Hardware/software codesign and system synthesis, pp. 2833. ACM, 2006.

[20] Isci, Canturk, and Margaret Martonosi. "Runtime power monitoring in high-end processors: Methodology and empirical data." In Proceedings of the 36th annual IEEE/ACM International Symposium on Microarchitecture, p. 93. IEEE Computer Society, 2003. 